\title{
Ensuring global access to COVID-19 vaccines: deployment strategies for refugees and migrants must not be forgotten
}

\author{
Danilo Buonsenso ${ }^{1,2,3}$ (1) Ulrich von Both ${ }^{4,5}$
}

Received: 25 April 2021 / Accepted: 20 May 2021 / Published online: 28 May 2021

○) Springer-Verlag GmbH Germany, part of Springer Nature 2021

World Health Organization (WHO) estimated 1 billion migrants in the world today of whom 258 million are international and 763 million internal migrants [1]. Migrants, refugees, and asylum seekers may be both more affected by and vulnerable to the spread of COVID-19. In the context of the COVID-19 pandemic, vulnerabilities of this group have exacerbated since many of them are living in crowded refugee camps or difficult housing conditions with limited means and opportunities to implement personal hygiene and social distancing. Consequently, COVID-19 outbreaks among this population can have devastating impact and have already been reported [2].

Recently, authors discussed in an important paper development and production, affordability, allocation and deployment of COVID-19 vaccines [3], highlighting important barriers and outlining necessary steps to implement in order to achieve global vaccine immunity. However, we feel that an important aspect relating to deployment of vaccines needs to be specifically highlighted: access to COVID-19 vaccination campaigns for migrants, refugees and asylum seekers.

Danilo Buonsenso

danilobuonsenso@gmail.com

Ulrich von Both

ulrich.von.Both@med.uni-muenchen.de

1 Department of Woman and Child Health and Public Health, Fondazione Policlinico Universitario A. Gemelli, Largo A. Gemelli 8, 00168 Rome, Italy

2 Dipartimento di Scienze Biotecnologiche di Base, Cliniche Intensivologiche e Perioperatorie, Università Cattolica del Sacro Cuore, Rome, Italy

3 Global Health Research Institute, Istituto di Igiene, Università Cattolica del Sacro Cuore, Rome, Italy

4 Division of Paediatric Infectious Diseases, Dr. von Hauner Children's Hospital, University Hospital, Ludwig Maximilians University, Lindwurmstrasse 4, 80337 Munich, Germany

5 German Centre for Infection Research (DZIF), Munich, Germany
Although sustainable health is universally considered a basic human right, access to healthcare systems varies and depends on several factors. Most countries have developed online platforms for booking appointments to rule out COVID-19 vaccination campaigns. This requires both the technical means and a regular registration status of the candidate booking the appointments. However, access to healthcare greatly varies across the globe. In general, legal status is the most important determining factor [4], with those considered "undocumented" having "a priori" limited access to basic services. The International Organization for Migration has estimated that $10-15 \%$ of the world's 214 million international migrants were undocumented [5], suggesting that millions of people worldwide may have difficulty in accessing the vaccine. To make things more complicated, refugees' and migrants' access to basic health services may be further impacted by their employment status [4]. Undocumented labor migrants may choose not to present to health care institutions because of fear of deportation [4]. Even with documented legal and employment status, other barriers may further limit access, such as language barriers, lack of accessible information channels, unawareness of entitlements by health care providers and beneficiaries, limitations of health staff expertise, and economic limitations [4].

These points highlight potential difficulties for providing equal access to SARS-CoV-2 vaccines for migrants and refugees. They need to be proactively addressed by institutions and policy-makers with dedicated plans, since most standard administrative procedures would not allow easy access to vaccination programs for migrants and refugees.

The first attempt to provide equity in SARS-CoV-2 vaccines access was represented by the COVAX program, one of three pillars of the Access to COVID-19 Tools (ACT) Accelerator, launched in April 2020 by the WHO, the European Commission and France in response to this pandemic (https://www.who.int/initiatives/act-accelerator/covax). The ACT aims to bring together governments, global health organizations, manufacturers, scientists, the private sector, 
civil society and philanthropy with the aim of providing innovative and equitable access to COVID-19 diagnostics, treatments and vaccines [6]. The COVAX pillar represents the last ACT pillar and specifically aims, as the most urgent point, on equitable and universal access to vaccines. Currently, this program is working primarily in distribution of enough vaccines in low-to-middle income countries (LMICs), although the program contains in its memorandum a specific section for fragile populations. The program, in fact, aims to develop a "humanitarian buffer, to be made available as a backstop mechanism to serve as a provider of last resort for if/when national, government-led planning and roll-out fail to reach certain high-risk populations in humanitarian settings. For example, populations living outside government-controlled areas could be served through the humanitarian buffer". However, the statement concludes by highlighting the potential challenges in implementing such a "buffer", explaining that "this would be implemented once all other options have been explored; and applicants would be asked to demonstrate a gap in coverage among relevant populations within the scope of this buffer" [7].

Currently, only few countries worldwide have active vaccination programs specifically including migrants and refuges. According to European Commission statistic data, there are currently around 2.6 million refugees in Europemostly settled in Germany, Spain, France, Italy and Greece. A Pew Research Center report estimated that as many as 4.8 million undocumented migrants were living in Europe in 2017-almost half of them in Germany. Here, refugees can access health care almost on the same basis as German citizens. Those who live in refugee centers are actually prioritized in the vaccination campaign. The same applies to asylum seekers who have seen their asylum request rejected. In Germany, these people receive a "tolerated status", Duldung, whereby they can access certain health care. This program also includes access to the COVID-19 vaccines. The service is recognized in Germany as an "emergency service" and thus allows access for migrants and refugees with costs covered by the State [8]. The German approach confirms that vaccinating undocumented people is potentially feasible. Outside Europe, positive experiences with active vaccination programs have been reported in Nepal, Jordan and Rwanda.

Other countries (in Europe Italy, France, UK, France, Belgium, Netherlands, Finland) have programs that include undocumented people, however on a practical day-to-day basis it is poorly understood how these people can access vaccination programs; and there are no official governmental data providing how many refugees and migrants have been immunized [8]. Conversely, while refugees and asylum seekers in Greece can in principle access vaccines, there is no clear provision for undocumented people, according to Villads Zahle from the European Council on Refugees and
Exiles (ECRE) [9]. Some government authorities, on the other hand, are working to return many asylum seekers from refugee camps to their respective countries of origin. Others have explicitly excluded non-resident foreigners from the national vaccination program.

A more feasible approach would be to actively engage national and international NGOs. These could be provided with vaccine vials and allowed to directly reach out to migrants and refugees administration of vaccinations, at least in the traceable and known refuge/migrant population. Here, standard prioritization approaches can be followed, first aiming at those at highest risks, such as the elderly and people with comorbidities, i.e., like Down syndrome and other congenital disorders [10].

Another critical obstacle in this process is the fear of undocumented refugees to be recognized when participating in a vaccination program, thus risking subsequent deportation. However, in the current pandemic scenario, having most people immunized, and particularly those living in fragile and crowded settings, is particularly important. In order to end the pandemic, it is a priority that all those eligible are vaccinated since otherwise there will always be a risk of new outbreaks. For these specific scenarios, NGOs or emergency vaccination services may provide immunization and a paper-based vaccine certificate and ask people if they would like to be registered on national registries; policymakers and institutions may agree to include an anonymous code for those who wish to not be registered.

A SARS-CoV-2 vaccine campaign focused on migrants and refuges would have a number of specific indirect benefits for children as well. Although the direct impact of the pandemic on children has been relatively mild, indirect effects like social restrictions and school closures continue to have a major impact on children's health and mental wellbeing [11]. The impact has been particularly devastating for fragile families unable to provide means for distance learning. Leaving entire communities of migrants and refuges unprotected would allow the virus to circulate and, indirectly, again impact on children's health. Also, while it is true that children mostly present with a mild-to-moderate clinical course of COVID-19, it is also evident that children from fragile backgrounds have a higher risk of developing more severe disease or the recently described post-COVID multisystem inflammatory syndrome in children (MIS-C) [12]. The need of protecting children, and particularly the most fragile, further highlights the importance of including migrants and refuges in SARS-CoV-2 vaccine programs.

In 2018, the WHO stated that "to achieve the vision of the 2030 agenda and the sustainable development goals, to leave no one behind, it is imperative that the health needs of refugees and migrants be adequately addressed" [3]. Thus, policies and prioritization mechanisms to ensure that migrants, refugees and asylum seekers are not left behind in 
deployment strategies for COVID-19 vaccines are urgently needed to prevent a "transmission of vulnerability" in this population. Mobile vaccine-clinics reaching these communities and one-dose COVID-19 vaccines allowing refrigeration for storage would be of great practical benefit to respective immunization campaigns in this group [1].

Funding No funds received.

\section{Disclosure}

Conflict of interest Nothing to declare.

\section{References}

1. Redditt V, Wright V, Rashid M, Male R, Bogoch I. Outbreak of SARS-CoV-2 infection at a large refugee shelter in Toronto, April 2020: a clinical and epidemiologic descriptive analysis. CMAJ Open. 2020;8(4):E819-24.

2. Wouters OJ, Shadlen KC, Salcher-Konrad M, Pollard AJ, Larson HJ, Teerawattananon Y, Jit M. Challenges in ensuring global access to COVID-19 vaccines: production, affordability, allocation, and deployment. Lancet. 2021. https://doi.org/10.1016/ S0140-6736(21)00306-8.

3. https://www.who.int/migrants/en/. Accessed 15 Feb 2021

4. https://www.who.int/migrants/publications/EURO-report.pdf. Accessed 15 Feb 2021

5. https://www.un.org/en/development/desa/population/publications/ pdf/policy/InternationalMigrationPolicies2013/Report\%20PDFs/ k_Ch_5.pdf. Accessed 15 Feb 2021
6. https://www.who.int/initiatives/act-accelerator/covax. Accessed 1 May 2021

7. https://www.gavi.org/covax-facility/protecting-human-rights. Accessed 1 May 2021

8. https://picum.org/covid-19-vaccines-undocumented-migrantseurope/. Accessed 1 May 2021

9. https://www.dw.com/en/refugees-and-undocumented-migrantsmust-be-vaccinated-ngos-warn/a-56664623. Accessed 1 May 2021

10. Kim-Hellmuth S, Hermann M, Eilenberger J, Ley-Zaporozhan J, Fischer M, Hauck F, Klein C, Haas N, Kappler M, Huebner J, Jakob A, von Both U. SARS-CoV-2 triggering severe acute respiratory distress syndrome and secondary hemophagocytic lymphohistiocytosis in a 3-year-old child with down syndrome. J Pediatric Infect Dis Soc. 2021;10(4):543-6. https://doi.org/10. 1093/jpids/piaa148.PMID:33188394;PMCID:PMC7717321.

11. Buonsenso D, Roland D, De Rose C, Vásquez-Hoyos P, Ramly B, Chakakala-Chaziya JN, Munro A, González-Dambrauskas S. Schools closures during the COVID-19 pandemic: a catastrophic global situation. Pediatr Infect Dis J. 2021;40(4):e146-50. https:// doi.org/10.1097/INF.0000000000003052 (PMID: 33464019).

12. Antúnez-Montes OY, Escamilla MI, Figueroa-Uribe AF, ArteagaMenchaca E, Lavariega-Saráchaga M, Salcedo-Lozada P, Melchior P, de Oliveira RB, Tirado Caballero JC, Redondo HP, Montes Fontalvo LV, Hernandez R, Chavez C, Campos F, Uribe F, Del Aguila O, Rios Aida JA, Buitrago AP, Betancur Londoño LM, Mendoza Vega LF, Hernández CA, Sali M, Higuita Palacio JE, Gomez-Vargas J, Yock-Corrales A, Buonsenso D. COVID-19 and multisystem inflammatory syndrome in Latin American children: a multinational study. Pediatr Infect Dis J. 2021;40(1):e16. https://doi.org/10.1097/INF.0000000000002949 (PMID: 33055501). 